\title{
Clinical Activity of Anti-HER2-Antibody Drug Conjugates in HER2-Mutated Metastatic Cancer Patients
}

\author{
Albert J. (AJ) Brougham 1 , Jeffrey Solzak ${ }^{1,2}$, Milan Radovich 1,2,3 \\ ${ }^{1}$ Indiana University School of Medicine, ${ }^{2}$ Indiana University School of Medicine, Department of \\ Surgery, ${ }^{3}$ Indiana University Health Precision Genomics Program
}

Background/Objective: Antibody drug conjugates (ADCs) deliver potent cytotoxic therapy in a highly targeted manner by binding cancer-specific cell surface antigens. HER2 is a receptor tyrosine kinase that is commonly amplified in breast and gastric cancers, but more recently has been shown to harbor gain-of-function activating mutations in several cancer types. Recent observations have suggested that HER2-ADCs may be viable therapies for patients that harbor these activating mutations. This research retrospectively explores the efficacy of HER2-ADCs in metastatic cancer patients in a real-world setting.

Methods: Patient information was gathered from the Precision Genomics program at IU Health, narrowed for specific mutation criteria (HER2 pathogenic mutation), cross referenced with OncoKB to legitimize oncogenicity, and selected for patients prescribed HER2-ADCs. For these patients, pre-treatment and mid-treatment CT-scans were analyzed following RECIST protocols. The primary outcomes were overall response rate (ORR), clinical benefit rate (CBR), and progression free survival (PFS). Secondary outcomes were to tabulate the frequency and clinical characteristics of HER2-mutated tumors.

Results: Out of 517 patients with somatic HER2 mutations, 60 patients had a pathogenic HER2 mutation. Of these 60 patients, the most common tumor types were $26.67 \%$ Breast and $11.67 \%$ Bladder/Urothelial. 11 of 60 patients were prescribed a HER2-ADC (Trastuzumab Emtansine = 10, Trastuzumab Deruxtecan $=1$ ). 8 of 11 patients were evaluable for response with RECIST criteria with 1 patient having a partial response, 4 patients having stable disease and 3 patients having progressive disease. $\mathrm{ORR}=13 \%, \mathrm{CBR}=63 \%$, Median PFS $=2.77$ months (95\% Cl: $2.15-$ 3.39 months).

Conclusion/Implications: To our knowledge, this is the first report of HER2-ADCs demonstrating clinical activity in HER2-mutated cancers across tumor types. Further clinical trials are ongoing that will validate these initial findings. 